\title{
Application of the CNC system based on PMAC in Laser Forming Machine
}

\author{
Chun Shang \\ Shenyang Aerospace University \\ Key Laboratory of Fundamental Science for National \\ Defence of Aeronautical Digital Manufacturing Process \\ Shenyang, China \\ shangchun163@163.com \\ Peng-fei Guo \\ Shenyang Aerospace University \\ Key Laboratory of Fundamental Science for National \\ Defence of Aeronautical Digital Manufacturing Process \\ Shenyang, China \\ guopengfei880614@126.com
}

\section{Guang Yang}

Shenyang Aerospace University

Key Laboratory of Fundamental Science for National Defence of Aeronautical Digital Manufacturing Process

Shenyang, China

yangguang@sau.edu.cn

\author{
Hong-you Bian \\ Shenyang Aerospace University \\ Key Laboratory of Fundamental Science for National \\ Defence of Aeronautical Digital Manufacturing Process \\ Shenyang, China \\ bianhongyou@sau.edu.cn

\section{Peng Qi} \\ Shenyang Aerospace University \\ Key Laboratory of Fundamental Science for National \\ Defence of Aeronautical Digital Manufacturing Process \\ Shenyang, China \\ dboyqi@163.com

\section{Lei Cai} \\ Shenyang Aerospace University \\ Key Laboratory of Fundamental Science for National \\ Defence of Aeronautical Digital Manufacturing Process \\ Shenyang, China \\ cailei198625@126.com
}

\begin{abstract}
With the research of the main structure of the CNC System, the design philosophy of the CNC System based on PMAC in Laser Forming Machine was put forward. This system takes PMAC as control model of CNC and PC as system supporting units, which is a double CPU CNC system- the CNC system model of "PC+PMAC". A lot of experiments have proved that this system can work steadily and it can meet the demand of laser forming machine.
\end{abstract}

Keywords- PMAC; CNC system; Laser forming machine

\section{INTRODUCTION}

The CNC laser machining technology is a advanced forming technology which gathers laser, mechanics, electronics and so on together. It is not only widely used in areas of heat treatment, surface cladding, cutting and welding of material, but its machining process is automatic. So it will be widely-used in the future ${ }^{[1]}$.The traditional laser forming machine is mainly equipped with special CNC system, but this system uses closed structural model, which restricts the system's scalability and flexibility. So it is difficult to integrate enterprise process methods experience with special technology into the control system, which abandons many functions of the CNC system and makes the cost much higher ${ }^{[2]}$. The open CNC system is recognized as owning an emerging construction pattern of the numerical control system, which has a lot of merits such as extendibility, reusability, probability, interchangeability ${ }^{[2]}$. In order to enable numerical control system of the laser forming machine to have the merits of the open numerical control system, this system uses the typical structure of the open numerical control system, namely "PC+PMAC" control structure. This paper mainly describes the structure of the open CNC system and analyses the construction of the open CNC system of laser forming machine and the software and hardware components in detail.

\section{THE OPEN CNC SYSTEM AND ITS STRUCTURE FORM}

IEEE standard defines that open CNC system can run on multiple platforms, can be operated by other systems and can provide a unified style of interaction ${ }^{[3]}$. At present, the open CNC system at home and abroad has four kinds of structural forms. They are as follows ${ }^{[4]}$ :

(1) The style of PC connecting to NC. This style means PC connects to the original CNC system transmitting information with serial communication. It comes true easily, but the original CNC system limits its functions, which contributes to the whole system responding slowly. As a result, it cannot become opened easily.

(2) The style of PC inserted into NC. This style means inserting NC into PC and they are connected by special bus. It transforms data fast, responses quickly and makes the most of the CNC system, but its front interface can connect to PC. In a word, this mode opens partly, but the core of NC is unopened, limiting its openness.

(3)The style of NC inserted into PC. The style recognizes $\mathrm{PC}$ as the core of the system, and card was inserted into the standard expansion slot of PC to realize the standard NC function. Therefore, the style makes it easy to realize the openness of the interface connecting the users 
and the communication of the main system, meanwhile the function of the system is guaranteed and the generality of the software is strengthened further.

(4) The style of NC full of software. This NC is the open CNC system in real sense, whose CNC interface board only should be inserted into the standard slots of PC. The PC we mentioned is the general kind which is not need to be reformed, the whole PC system is extended from PC. Expect servo driving and external I/O interface, all functions are finished by software which are general and flexible in program processing. As a result, it makes complete openness a reality. On the whole, the open CNC system based on PC and multi-axis controller is a better one, which can put system platform on the basis of PC microcomputer software to make the most of the existing hardware and software resources and make the development of system simple and perform reliably. PMAC, which is a multi-axis controller, is widely used in the open CNC system in the industrial fields at home and abroad ${ }^{[5]}$.

\section{THE OVERALL DESIGN OF THE OPEN CNC SYSTEM BASED ON PMAC IN LASER FORMING MACHINE}

\section{A. The control requirements of the CNC system in laser forming machine}

The basic principle of the laser forming machine is that the tool focuses laser with enough power(or energy) on metal powder which is provided by powder feeder to melt the metal powder and change the metallographic structure so that it achieves the goal of forming ${ }^{[6]}$. The laser forming machine has four transmission shafts containing $\mathrm{X}$-axis, $\mathrm{Y}$-axis, Z-axis and the spindle of powder feeding device. The X,Y,Z-axes rotates simultaneously to control the position of the worktable so that the design drawings of the forming part can be realized, while the spindle leads powder disc rotating at a constant speed automatically. The $\mathrm{X}, \mathrm{Y}, \mathrm{Z}$-axes are driven by the ball screws connecting to the AC servo motor through the synchronous toothed belts, while the spindle is driven by the stepping motor with worm gear reducer. The mechanism and coordinate position of the machine are displayed by the figure 1 as follows:

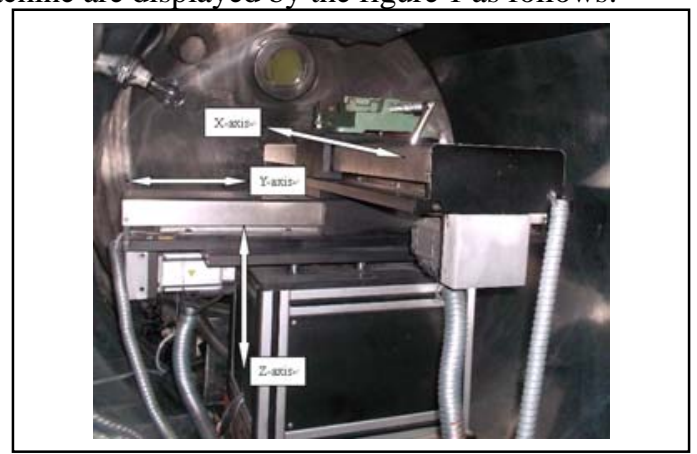

Figure 1. The structure and the coordinate system of the machine
B. The hardware design of the CNC system in the laser forming machine .

According to the control requirements of the system, the scheme of the hardware-structure design is shown in figure 2.

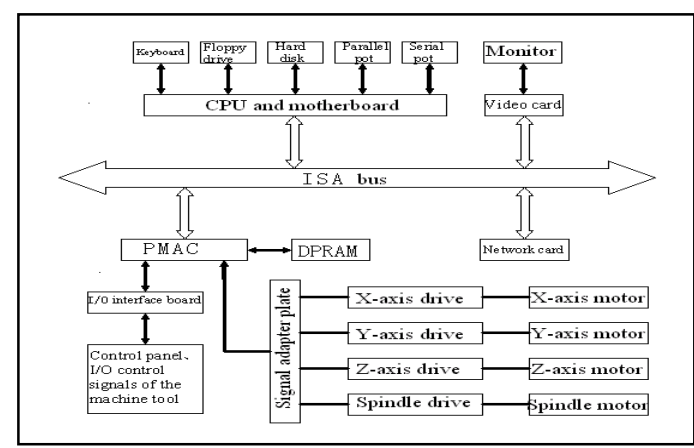

Figure 2. The structure of the hardware of the CNC system

The system has a structure form of the upper and hypogynous machine based on "PC+PMAC motion control card". The upper machine using PC mainly controls the system, while the hypogynous machine using PMAC motion control card -PMAC 2-LITE completes the real-time control over the motion of the X,Y,Z-Axis and spindle of powder feeding device and the switches of the control panel.

\section{The software design of the CNC system in the laser forming machine.}

\section{- The overall design of the software structure}

The software of CNC system which gained the functions of pipeline, detection and control, is the control center in the whole CNC system. It cannot run normally without the software of the CNC system ${ }^{[7]}$. This system software is developed on the software platform based on Windows and regards $\mathrm{VC}++6.0$ complier as the basic tool of the software development and debugging programs ${ }^{[8]}$. The software structure is shown in figure 3.

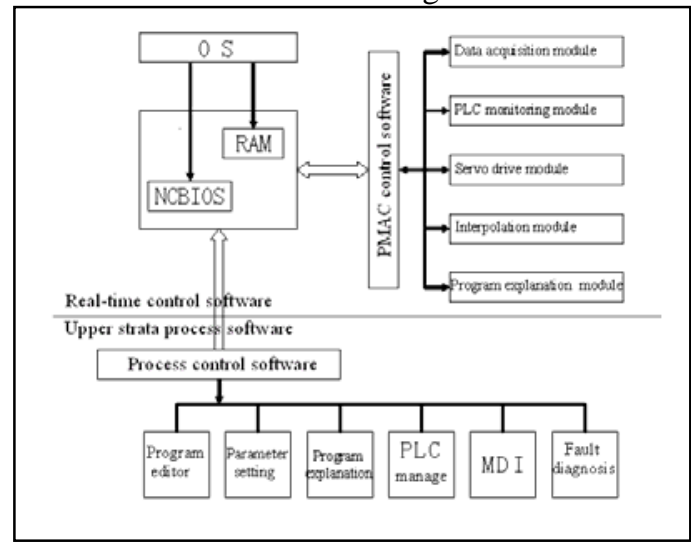

Figure 3. The software structure of the CNC system.

The modular design methods are used in the system software. These methods make the software open. As a 
result, every module not only run continuously but run independently. The main modules are as follows:

(1)Data acquisition module. This module is used to dispatch the resources of the management center and each task process dynamically, collecting data from I/O cards or sending data to $\mathrm{I} / \mathrm{O}$ cards at any time. In coordination with the system, each module runs highly efficient and safely.

(2)PLC control module. It mainly displays the actual position and the given position of each coordinate axis and the system processing program, alarm information and so on at any time.

(3)Servo driving module. It mainly realizes various sports of the working table, such as progressive movement, continuous movement, linear interpolation and circular interpolation, etc. It also adjusts speed in the processing to improve the efficiency.

(4)Interpolation module. This module controls the accuracy of each axis to ensure the machining accuracy of machine tools.

(5)Machining program module. It mainly completes interpretation program, decoding program, transforming program before machining, budgeting and processing. In order to check the correctness of the processing program, the module can make a motion stimulation to the processing program.

(6)Displaying module. It feedbacks the processing programs, the motion track of the working table, the running state of the system to the operator.

(7)The fault diagnosing module. This module analyses and diagnoses the faults of the system's processing and feedbacks the information to the operator.

- The PLC program design of PMAC

The PLC program design of PMAC uses structured design method, whose structure contains selection structure, sequence structure and loop structure. Before running a PLC program, the finished PLC program is loaded into the PLC program buffer of PMAC, closing the original buffer. The buffer must be closed after running the program to the end. The basic form of the PLC program of this CNC system is shown as follows:

$$
\begin{array}{ll}
\text { OPEN PROR } & \text { XX } \\
\text { bLEAR } & \text { program // open the program } \\
\text {..... } & \text { code in buffer } \\
\text { CLOSE } & \text { buffer }
\end{array}
$$

\section{CLOSE}

program

- The design of the man-machine interaction interface

The man-machine interaction interface links man and the computer together. The operators input and modify control parameter to PC through the interface and send various control instructions; PC also displays running state of the system and sends alarm information to the operator through the interface. When developing CNC system of the laser forming machine, all kinds of practical function interfaces can be designed according to functional requirements of the $\mathrm{CNC}$ system. The main interfaces are as follows:

1) Programming interface

Programming interfaces are shown in fig 4.This interface is mainly used to edit the program to run, then loads the finished programs into PMAC so that the CNC system can run the program.

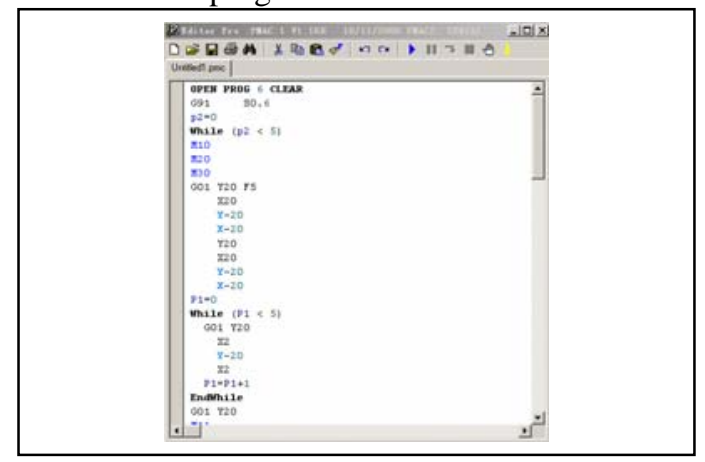

Figure 4. The interface of program edit

2) The manual interface

The manual interface is shown in fig 5.The interface is mainly used to debug the laser machine and adjust the working table. The buttons in the top of the status bar are used to control switches, while the buttons in the below are used to adjust the working table and display the position and speed of the working table.

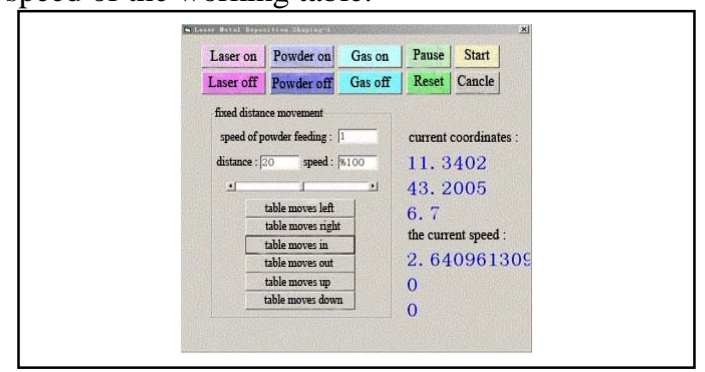

Figure 5. The manual interface

\section{THE END}

The powerful functions of PMAC and the excellent openness and scalability of the software and hardware are verified in detail by researching the open CNC system and introducing its applications in laser forming machine. The CNC system based on "PC+PMAC" is flexible in practice, which can be configured flexibly according to the specific circumstance. PMAC, which is a widely used motion controller ,is very popular at home and abroad.

\section{REFERENCES}

[1] ZHANG Guoshun. "Modern laser manufacturing technology". Beijing Chemical Industry Press,2006:7-15.

[2] ZHOU Jianzhong, LIU Huixia. "Laser rapid manufacturing technology and Application” .Beijing Chemical Industry Press,Feb,2009,pp.16-19,31-32. 
[3] SUN Bin,YANG Ruqing. "Research present situation and development trend of the CNC system based on PC”.Mechine Tool \& Hydraulics,no.2,April 2001.

[4] LI Aiping,ZHANG Jianguo."Study of the open architecture numerical control system of the NC- embedded PC structure”.Modular Machine Tool \& Automatic Manufacturing Technique,Dec 2001,pp.1-2.

[5] WANG Yihong,CHEN Zhitong."Application of Handwheel for CNC Machine Based on PMAC”.MECHANICAL ENGINEER.July 2005.
[6] SHAO Dan,HU Bing,ZHENG Qiguang."Laser advanced manufacturing and equipment integration”. Beijing Science Press. Oct 2009,pp.28-30.

[7] QIAN Junhan.”Design and realization of the open CNC system based on PMAC”.Modular Machine Tool \& Automatic Manufacturing Technique,April 2008,pp.20.

[8] DING Shiyan,HAN Hong,ZHU Fangzhou.etc "Research of the Software Platform of OpenType NC System".Machinery \& Electronics,Feb 2002,pp.2 